\title{
Use of mifepristone for termination of intrauterine fetal demise (IUFD) in previously scarred uterus in later half of pregnancy (>20 weeks)
}

\author{
R. Arora, P. B. Patel*, A. Dabral, M. Sachdeva, P. Arya
}

Department of Obstetrics and Gynecology, Vardhman Mahavir Medical College and Safdarjung hospital, New Delhi, India

Received: 03 June 2018

Accepted: 16 June 2018

*Correspondence:

Dr. P. B. Patel,

E-mail: preetikgmc@gmail.com

Copyright: (c) the author(s), publisher and licensee Medip Academy. This is an open-access article distributed under the terms of the Creative Commons Attribution Non-Commercial License, which permits unrestricted non-commercial use, distribution, and reproduction in any medium, provided the original work is properly cited.

\begin{abstract}
Background: Mifepristone has the potential to be used as an agent for induction of labour by increasing the uterine contractility and increasing the sensitivity of uterus to prostaglandins. The present study is an endeavor to study the effect of mifepristone alone to induce labour in scarred uterus and its risk benefit ratio.

Methods: Total 39 patients with IUFD and previous uterine surgery were included in the study after their informed consent. All women in the study were given Tablet Mifepristone $200 \mathrm{mg}$ orally, thrice a day, maximum 6 doses (Max $-1200 \mathrm{mg}$ ) over a duration of 48 hours. Patients were monitored for vitals, the uterine contractions and any bleeding per vaginum. Next dose of drug was omitted if sufficient uterine contractions or cervical dilatation $\geq 2.5 \mathrm{~cm}$ achieved. Patients were shifted to the labour room after onset of active labour. Labour was augmented with oxytocin wherever required.

Results: spontaneous labour occurred in $74.3 \%$ (29/39) women while operative (cesarean/ hysterotomy) delivery occurred in $17.9 \%$ (07/39). Mean induction (first dose of mifepristone) to delivery interval was $51.5 \mathrm{hrs}$ in second trimester while $59.8 \mathrm{hrs}$ in third trimester women. Oxytocin augmentation was done in 8 (20.5\%) women.

Conclusions: The potential advantage of mifepristone over prostaglandins and oxytocin, is mainly in situations where they are contraindicated (i.e., scarred uterus). In this study authors found that with mifepristone only regimen is quite safe and effective, inducing spontaneous labour in $74.3 \%$ (29/39) women with IUFD and in reducing the operative (cesarean/ hysterotomy) delivery (17.9\%).
\end{abstract}

Keywords: Induction of labour, IUFD, Mifepristone, Scarred uterus

\section{INTRODUCTION}

Intrauterine fetal demise is a common problem with 1 in 200 babies born dead. ${ }^{1}$ A death that occurs prior to 20 weeks gestation is usually classified as a spontaneous miscarriage and those occurring after 20 weeks constitute fetal demise or stillbirth. ${ }^{2}$ When IUFD occurs, spontaneous expulsion may take several weeks. Psychological distress and risk of complications like DIC may compel most clinicians to induce fetal expulsion when possible. Moreover, these days we are also encountering increasing number of indications for termination of pregnancy owing to greatly improved detection of antenatal malformations. Since the number of cesarean section is also on rise, there is increasing likelihood of medical indication for termination of pregnancy in patients with previous cesarean section. Induction of labour in women with previously scarred uterus is not easy as it can lead to life threatening events like rupture uterus, retained placenta and excessive bleeding. So, induction in these women requires tertiary setup for close monitoring, diagnosis and treatment of 
hyperstimulation and uterine rupture, availability of blood transfusion and surgical intervention.

Main aim of authors in present study was termination in an intra-uterine fetal death to achieve uncomplicated vaginal delivery, which is relatively less problematic in an unscarred uterus than scarred uterus. Though various modalities of termination of pregnancy have been suggested, there are very limited studies to describe the method of termination in late IUFD in cases where prostaglandins are contraindicated, especially in cases with previous cesarean section and we need a method that does not increase the uterine contractility per say but induces labour naturally. Mifepristone is a new class of pharmacological agents which antagonize the action of progesterone. It is 19 nor steroid which has greater affinity for progesterone receptors than does progesterone itself thus blocking the action of progesterone. It has rapid absorption and long half-life of 25-30 hrs. ${ }^{3}$ Thus, mifepristone has the potential to be used as an agent for induction of labour by increasing the uterine contractility and increasing the sensitivity of uterus to prostaglandins. ${ }^{4}$

The present study is an endeavour to study the effect of mifepristone alone to induce labour in scarred uterus and its risk benefit ratio.

\section{METHODS}

The study was a Prospective clinical study, conducted in the Department of Obstetrics and Gynecology VMMC and Safdarjung hospital, New Delhi, comprising of 39 antenatal women with previous uterine surgery.

\section{Inclusion criteria}

- Women with ultrasonically confirmed singleton pregnancy with intra- uterine fetal death or gross congenital malformation requiring termination of pregnancy, at gestational age more than 20 weeks

- with absence of any clinical features of imminent labour.

\section{Exclusion criteria}

- All patients who had any contraindication to vaginal delivery (multiple pregnancy, non-vertex presentation), any untreated bleeding or coagulation disorder

- known hypersensitivity to mifepristone, sepsis, or medical problems like, impaired renal, hepatic or adrenal function.

All women underwent detailed history, clinical examination, counseling, informed consent and initial investigations like hemogram, Blood group, Urine Routine and microscopy, Liver and Kidney function test and coagulation profile. Ultrasound was done to confirm the IUFD.
Patients were given Tablet Mifepristone $200 \mathrm{mg}$ orally, thrice a day, maximum 6 doses (Max -1200 mg) over a duration of 48 hours. Patients were monitored for vitals, the uterine contractions and any bleeding per vaginum. Next dose of drug was omitted if sufficient uterine contractions or cervical dilatation $\geq 2.5 \mathrm{~cm}$ achieved. Patients were shifted to the labour room after onset of active labour. Labour was augmented with oxytocin wherever required.

Patients who did not had uterine contractions or did not progressed into labour within 72 hrs of onset of induction were regarded as failures. Patients were observed for 24 hours following delivery, for evidence of any complication like PPH, Infection, DIC, retained placenta.

In present study primary outcome was successful vaginal delivery within 72 hours of onset of induction \& Secondary outcome was to find any evidence of scar dehiscence and scar rupture.

\section{RESULTS}

Total 39 pregnant women with previous uterine surgery and IUFD were given mifepristone regimen as described above. As depicted in Table 1, mean age of the women studied were 29.3 yrs. Out of $39,34(87.1 \%)$ women had previous one cesarean section, $4(10.2 \%)$ had previous two cesarean section and one $(2.5 \%)$ woman had previous myomectomy. $16(41.02 \%)$ women were in second trimester (20-28 weeks) while 23 (58.9\%) women were in third trimester (29-40 weeks).

Table 1: Characteristics of studied women.

\begin{tabular}{|ll|}
\hline Total no of patients & 39 \\
\hline Prev 1 LSCS & $34(87.1 \%)$ \\
\hline Prev 2 LSCS & $04(10.2 \%)$ \\
\hline Prev Myomectomy & $01(2.5 \%)$ \\
\hline Mean age of patients & 29.3 years \\
\hline Period of gestation & \\
\hline Second trimester (20-28 week) & $16(41.02 \%)$ \\
\hline Third trimester (29-40 week) & $23(58.9 \%)$ \\
\hline
\end{tabular}

As shown in Table 2, spontaneous labour occurred in $74.3 \%$ (29/39) women while operative (cesarean/ hysterotomy) delivery occurred in $17.9 \%$ (07/39). In operative delivery group, all except one operation was performed for failed induction. In one case cesarean was done on suspecting scar tenderness but intraoperatively no scar dehiscence was seen. In three $(07.6 \%)$ women vaginal delivery resulted after mechanical (balloon) dilatation after waiting for $48 \mathrm{hrs}$ of completion of six doses of mifepristone. Mean induction (first dose of mifepristone) to delivery interval was $51.5 \mathrm{hrs}$ in second trimester while 59.8 hrs. in third trimester women (table $3)$. Oxytocin augmentation was done in 8 (20.5\%) women. $26(66.6 \%)$ women received all 6 doses of mifepristone before they progressed into active labour. 
Overall, mean dose of mifepristone required to induce labour was $1040 \mathrm{mg}$.

Table 2: Outcome measurements of the induction of labour.

\begin{tabular}{|l|l|}
\hline Outcome & No. of patients (\%) \\
\hline Spontaneous labour & $29(74.3 \%)$ \\
\hline Cesarean section/ hysterotomy & $07(17.9 \%)$ \\
\hline Mechanical dilatation (balloon) & $03(7.6 \%)$ \\
\hline Complications & $3(07.6 \%)$ \\
\hline Fever & 0 \\
\hline Dehiscence/rupture & 0 \\
\hline PPH/ adherent placenta & 0 \\
\hline Infection/ chorioamnionitis & 0 \\
\hline Hyperstimulation & $8(20.5 \%)$ \\
\hline Oxytocine augmentation & \\
\hline Dose requirement & 2 \\
\hline $400 \mathrm{mg}$ & 3 \\
\hline $600 \mathrm{mg}$ & 1 \\
\hline $800 \mathrm{mg}$ & 6 \\
\hline $1000 \mathrm{mg}$ & $26(66.6 \%)$ \\
\hline $1200 \mathrm{mg}$ & 1040 \\
\hline Mean dose (mg) & \\
\hline
\end{tabular}

Maternal tolerance was good. No case of hyperstimulation/ rupture occurred. No uterine bleeding/ PPH/ adherent placenta occurred during observation period. Three $(07.6 \%)$ women developed mild fever during induction which subsided after giving antipyretics. No women developed intrapartum or post-partum infection.

Table 3: Induction - delivery interval.

\begin{tabular}{|ll|}
\hline Induction & Delivery interval \\
\hline Second trimester patients & $51.5 \mathrm{hrs}(41.2-68.4 \mathrm{hrs})$ \\
\hline Third trimester patients & $59.8 \mathrm{hrs}(45.4-72.0 \mathrm{hrs})$ \\
\hline
\end{tabular}

\section{DISCUSSION}

Mifepristone has been used successfully for first trimester abortion but its use to induce labour in late second and third trimester IUFD was first done by Cabrol in an open pilot study in $1985 .{ }^{5}$ Later in 1990, he did a double-blind placebo controlled multicentric study involving 94 women with IUFD to demonstrate the efficacy of mifepristone in a dose of $200 \mathrm{mg}$ three times a day, to induce labour. ${ }^{6} \mathrm{He}$ found that labour occurred in 63\% (29 of 46) patients in the mifepristone group compared to only in $17.4 \%$ in the placebo group $(\mathrm{p}<0.001)$.

Frydman and C Lelaidier used $200 \mathrm{mg}$ mifepristone or placebo for induction of labour in 120 women at term gestation on days 1 and 2 of a 4-day observation period, with labor induction planned for day $4.55 .3 \%$ subjects treated with mifepristone and ten $(17.8 \%)$ in the control group entered spontaneous labor $(\mathrm{P}<0.001){ }^{7}$ Patients who delivered vaginally needed a much lower amount of oxytocin when mifepristone had been given, and the mean time interval between day 1 of the survey and the onset of labor was also significantly shorter in this group.

Later, Lelaidier et al conducted a prospective doubleblind placebo-controlled trial to use mifepristone $200 \mathrm{mg}$ on day 1 and 2, to induce labour in women with previous cesarean section and found it to be safe and efficient agent for induction in women with previous cesarean section. ${ }^{8}$ They found that onset of labour occurred in $69 \%$ of patients in mifepristone group compared to only $12 \%$ in placebo group $(\mathrm{p}<0.01)$. Induction to onset of labour interval was $60 \mathrm{hrs} 35 \mathrm{~min}$ in study group and $82 \mathrm{hrs} 50$ min in placebo group $(\mathrm{p}<0.01)$.

Since then various studies have been done to evaluate the efficacy of mifepristone as an inducing agent in women with live term pregnancies either alone or in combination with prostaglandin analogues but none used mifepristone alone to induce labour in women with IUFD and scarred uterus.

Ahuja et al conducted a study comparing mifepristone alone in a dose of $200 \mathrm{mg}$ three times a day for two days (group 1) with combination of mifepristone followed by misoprostol (group 2) for induction of labour in women with term IUFD. In mifepristone alone group 19 patients with previous cesarean were included. ${ }^{9}$ The success rate was $90 \%$ in group I and $96.6 \%$ in Group II. The mean induction to delivery interval was $48.63 \pm 25.1 \mathrm{hrs}$ in group I while it was $68.87 \pm 21.1 \mathrm{hrs}$ in group II. $3.2 \%$ of patients in group I had side effects while $70 \%$ of patients in group II had side effects in the form of nausea, vomiting, fever and shivering.

In present study also spontaneous labour occurred in $74.3 \%$ women which is quite close to the success rate in studies by Cabrol, Frydman and Leiaidier but very less compared to study by Ahuja et al. ${ }^{6-9}$. Mean induction (first dose of mifepristone) to delivery interval was 51.5 hrs in second trimester which is quite close to finding of Ahuja et al but $59.8 \mathrm{hrs}$ in third trimester compared to $68.87 \pm 21.1$ hrs in study by Ahuja et al. ${ }^{9}$

In all these studies, clinicians have used different dose schedule of mifepristone i.e. optimal dose and frequency of mifepristone has not been defined which could be the reason for the difference in the results.

In 2005 Berkane et al conducted a double-blind placebocontrolled dose finding study in 346 women with live term pregnancies. ${ }^{10}$ They have used 50, 100, 200, 400 and $600 \mathrm{mg}$ of mifepristone or placebo. The main end point for efficacy was no of patients in whom labour occurred between 12, 45 and 54 hrs. The interval between treatment and labour onset, and between treatment and delivery, tended to be shorter as the mifepristone dose increased. The difference was significant between $600 \mathrm{mg}$ and placebo. Duration of labour also tended to be increase with the mifepristone dose. In present study 
authors used the doses protocol used by Cabrol (200 mg of mifepristone three times a day for two days) and found that $66.6 \%$ women needed $1200 \mathrm{mg}$ (6 doses) of mifepristone and mean dose required was $1040 \mathrm{mg}^{6}$

In Cochrane systematic review study (2009) "Mifepristone for induction of labour" (which included 10 trials on 1108 women), Hapangama et al found that mifepristone treated women were more likely to have favorable cervix at $48 \mathrm{hrs}$ compared to placebo group [risk ratio (RR) $2.41,95 \%$ confidence intervals (CI) 1.70 to 3.42] and this effect persisted at 96 hours (RR 3.40, $95 \%$ CI 1.96 to 5.92$){ }^{4}$

They were less likely to need augmentation with oxytocin (RR 0.80 , 95\% CI 0.66 to 0.97). Mifepristone treated women were less likely to undergo caesarean section (RR $0.74,95 \%$ CI 0.60 to 0.92 ) but more likely to have an instrumental delivery (RR 1.43 , 95\% CI 1.04 to 1.96). Athawale et al in their study on 100 women with term live pregnancy used mifepristone $200 \mathrm{mg}$ PO and assessed bishops score $24 \mathrm{hrs}$ and $48 \mathrm{hrs}$ of induction and found that rate of successful vaginal delivery was $76 \%$ in study group compared to only $36 \%$ in control group receiving placebo. ${ }^{11}$

\section{CONCLUSION}

This study was conducted to evaluate the efficacy and safety of medical management using mifepristone alone for induction of labour in previously scarred uterus. In this study authors found that with mifepristone only regimen is quite safe and effective, inducing spontaneous labour in $74.3 \%$ (29/39) women and reducing the operative (cesarean/ hysterotomy) delivery (17.9\%).

Since this was a pilot study where mifepristone has been used successfully for termination of pregnancy with IUFD where other methods of termination are risky, authors need a larger, placebo-controlled study to prove the efficiency and safety of mifepristone alone as an inducing agent in previously scarred uterus.

The potential advantage of mifepristone over prostaglandins and oxytocin, mainly in situations where they are contraindicated (i.e., scarred uterus) has been studied, but requires more studies.

Even though mifepristone is expensive, as it can be administered on outpatient basis and significantly less need for hospital admission, thereby reducing the overall cost. Hence mifepristone provides an interesting new alternative to classic uterotonic agents used for induction of labor at term in previously scarred uterus and can be considered by the obstetricians as a simple and safe method of labor induction.
Funding: No funding sources

Conflict of interest: None declared

Ethical approval: The study was approved by the Institutional Ethics Committee

\section{REFERENCES}

1. Confidential Enquiry into Maternal and Child Health (CEMACH). Perinatal Mortality 2007: United Kingdom. CEMACH: London, 2009. Available at: http://www.cmace.org.uk/getattach ment/1d2c0ebcd2aa-4131-98ed-56bf8269e529/PerinatalMortality2007.aspx.

2. Belkin T, Wilder J. Management Options for Women with Midtrimester Fetal Loss: a case report. J Midwifery Women Health. 2007;52(2):164-7.

3. Heikinheimo O. Clinical pharmacokinetics of mifepristone. Clin Pharmacokinet. 1997;33:7-17.

4. Hapangama D, Neilson JP. Mifepristone for induction of labour. Cochrane Database Syst Rev 2009;(3): CD002865.

5. Cabrol D, Bouvier D'Yvoire M, Mermet E, Cedard L, Sureau C, Baulieu EE. Induction of labor with mifepristone (RU 486) after intrauterine fetal death. Lancet 1985;2:1019.

6. Cabrol D, Dubois C, Cronje H, Gonnet J M, Guillot M, Maria B. Induction of labor with mifepristone (RU 486) in intrauterine fetal death. Am J Obstet Gynecol. 1990;163(2):540-2.

7. Frydman R, Lelaidier C, Baton -Saint-Mleux C, Fernandez H, Vial M, Bourget P. Labor induction in women at term with mifepristone (RU 486): A doubleblind, randomized, placebo-controlled study. Obstet Gynecol. 1992;80(6):972-5.

8. Lelaidier C, Baton C, Benifla JL, Fernandez H, Bourget P, Frydman R. Mifepristone for labour induction after previous caesarean section. Int J Obstet Gynaecol. 1994;101(6):501-3.

9. Ahuja N, Dahiya P. A comparative study of mifepristone alone versus mifepristone and misoprostol for induction of labor in intrauterine fetal death. Indian J Obstet Gynecol Res. 2016;3(4):348-351

10. Berkane N, Verstraete L, Uzan S, Boog G, Maria B. Use of mifepristone to ripen the cervix and induce labor in term pregnancies. A J Obstet Gynecol. 2005;192(1):114-20.

11. Athawale R, Acharya N, Samal S, Hariharan C. Effect of mifepristone in cervical ripening for induction of labour. Int J Reprod Contracept Obstet Gynecol. 2013;2(1):35-8.

Cite this article as: Arora R, Patel PB, Dabral A, Sachdeva M, Arya P. Use of mifepristone for termination of intrauterine fetal demise (IUFD) in previously scarred uterus in later half of pregnancy (>20 weeks). Int J Reprod Contracept Obstet Gynecol 2018;7:2668-71. 